\title{
Clinical Evidence for Adenoma-Carcinoma Sequence in Primary Retroperitoneal Mucinous Cystadenocarcinoma
}

\author{
Atsuko Taga ${ }^{1}$, Yukiyasu Sato ${ }^{1 *}$, Shota Kanbayashi ${ }^{1}$, Yasufumi Ri ${ }^{1}$, Ikuko Emoto ${ }^{1}$, Shunsuke Maruyama ${ }^{1}$, \\ Tomoyuki Shirase ${ }^{2}$ \\ ${ }^{1}$ Department of Obstetrics and Gynecology, Otsu Red Cross Hospital, 1-1-35 Nagara, Otsu 520-8511, Shiga, Japan \\ ${ }^{2}$ Department of Pathology, Otsu Red Cross Hospital, 1-1-35 Nagara, Otsu 520-8511, Shiga, Japan
}

*Corresponding Author: Yukiyasu Sato, Department of Obstetrics and Gynecology, Otsu Red Cross Hospital, 1-1-35 Nagara, Otsu 520-8511, Shiga, Japan, Tel: 81-77-522-4131; E-mail: yukiyasu@,kuhp.kyoto-u.ac.jp

Received: 23 February 2018; Accepted: 09 March 2018; Published: 12 March 2018

\begin{abstract}
Background: Adenoma-borderline tumor-carcinoma sequence, which was substantiated in the pathogenesis of ovarian mucinous carcinoma, could be extrapolated to that of primary retroperitoneal mucinous cystadenocarcinoma. Nevertheless, direct evidence is still insufficient due to its rarity.
\end{abstract}

Case: A 34-year-old Japanese woman with huge unilocular retroperitoneal cyst $(16 \times 8 \mathrm{~cm})$ refused surgery. The cyst was gradually shrunken, measuring $10 \times 4 \mathrm{~cm}$ at the age of 40 . One year later, however, remarkable enlargement of the cyst $(17 \times 14 \mathrm{~cm})$ with the emergence of three mural nodules was noted. The cyst was surgically removed intact. Histologically, a benign single layer of mucin-producing epithelial cells lined most of the cystic wall with focal microinvasive adenocarcinoma localized to the bases of mural nodules.

Conclusion: Primary retroperitoneal mucinous cystadenocarcinoma could arise from the pre-existing benign mucinous cystadenoma.

Keywords: Apparent Diffusion Coefficient Mapping; Diffusion-Weighted Imaging; Magnetic Resonance Imaging; Retroperitoneal Mucinous Cystadenoma; And Retroperitoneal Mucinous Tumor

\section{Introduction}

The retroperitoneum is an area where various soft tissue tumors could arise from the inherent structures, including muscle, nerve, and vascular tissue. Since surface epithelium is not normally present in the retroperitoneum, primary 
retroperitoneal mucinous tumor is a rare disease. Four major hypotheses have been postulated regarding possible origin of primary retroperitoneal mucinous tumors: ectopic ovarian tissue [1], retroperitoneal primary teratoma derived from displaced germ cells [2], intestinal duplication [3], and coelomic metaplasia [4]. Among them, the most favored is the last hypothesis, in which the mucinous tumor arises from invagination of the peritoneal mesothelial layer that undergoes mucinous metaplasia with cystic formation. This process is reminiscent of widely believed theory that ovarian epithelial neoplasms are originated from the metaplastic changes that take place in the inclusion cysts formed by the invaginations of ovarian surface mesothelium (so-called "ovarian surface epithelium") $[5,6]$. Although majority of primary retroperitoneal mucinous tumors grow as a unilocular cyst [7] in contrast to ovarian mucinous tumors that usually give a multilocular appearance with numerous septations, retroperitoneal and ovarian mucinous tumors could share similar pathogenesis.

As is the case in ovarian mucinous tumors, primary retroperitoneal mucinous tumors are divided into three categories: mucinous cystadenomas, mucinous borderline tumors, and mucinous cystadenocarcinomas. In the ovary, benign mucinous epithelia and/or mucinous borderline tumors coexist in invasive primary ovarian mucinous carcinoma in majority of the cases [8,9]. Moreover, mutation rates of oncogenes such as KRAS are similar among benign, borderline and malignant mucinous tumors [10]. These morphologic and genetic findings strongly support the idea that mucinous carcinomas arise from pre-existing benign and/or borderline mucinous tumors (i.e., adenoma-borderline tumor-carcinoma sequence). Although this sequence could be extrapolated to the case of primary retroperitoneal mucinous adenocarcinoma, direct evidence supporting this idea is still insufficient due to the rarity of the disease.

Here, we present a case of primary retroperitoneal mucinous cystadenocarcinoma that could lend evidence for the presence of adenoma-borderline tumor-carcinoma sequence in primary retroperitoneal mucinous tumor.

\section{Case}

A 34-year-old Japanese married woman, gravida 0, with a past history of appendectomy in her childhood received her first gynecological examination in the neighboring hospital. Multiple uterine fibroids and a unilocular cyst measuring $16 \times 8 \mathrm{~cm}$ in the right upper abdomen were detected by ultrasonography. In magnetic resonance imaging (MRI), the unilocular cyst was located in the retroperitoneal space and was apart from bilateral ovary. The cyst content showed low signal intensity on T1-weighted image (T1WI) (Figure 1A) and high intensity on T2WI (Figure 1D), which was a characteristic pattern of serous fluid. From these findings, the primary diagnosis was retroperitoneal Mullerian cyst [11]. Although the serum tumor markers examined were all within the normal range (CEA $0.2 \mathrm{ng} / \mathrm{ml}, \mathrm{CA} 19-914 \mathrm{IU} / \mathrm{ml}, \mathrm{CA} 1259 \mathrm{IU} / \mathrm{ml}$ ), the tumor markers in the aspirated content fluid were markedly high (CEA $730 \mathrm{ng} / \mathrm{ml}$, CA19-9 $25167 \mathrm{IU} / \mathrm{ml}$ ). The patient consistently refused to undergo operation, which was strongly recommended by the attending physicians. Afterwards, the patient was followed up every 6 months, monitoring the tumor size and being alert for potential emergence of mural nodules. The size of the tumor was gradually decreased and the serum tumor markers remained within the normal ranges. 

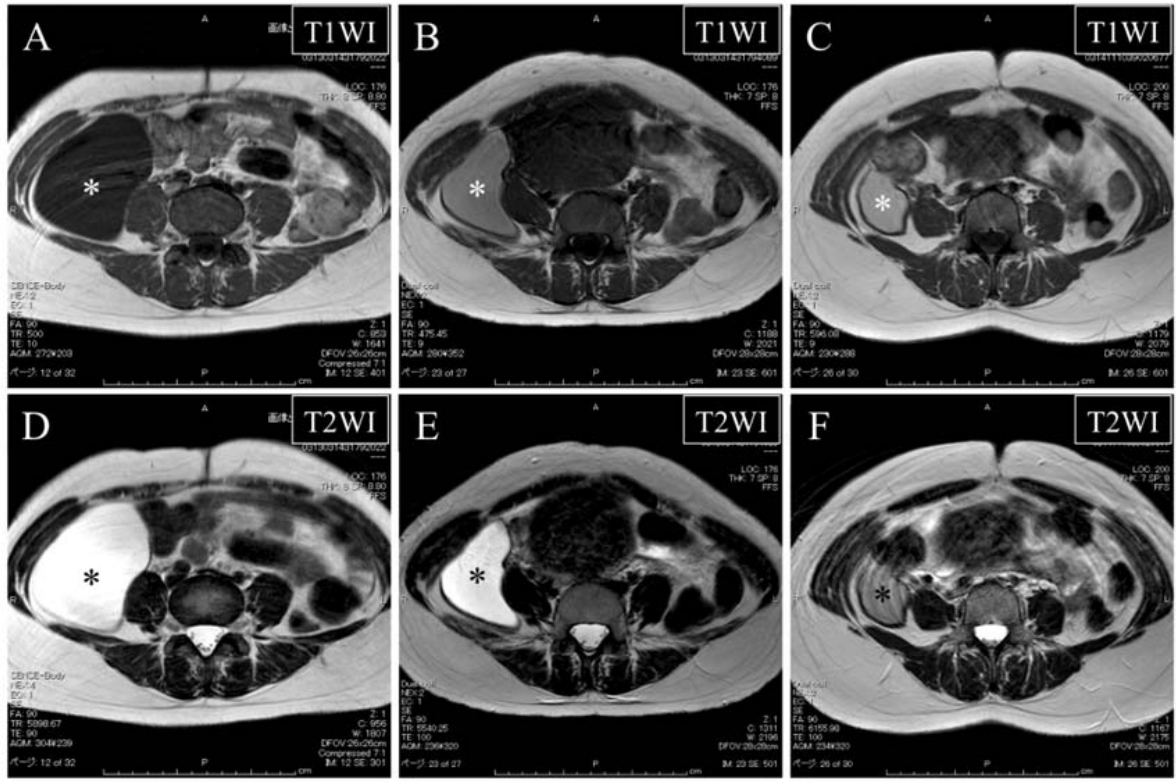

Figure 1: Magnetic resonance images at the ages of 34 (A and D), 38 (B and E), and 40 (C and F) showing gradual shrinkage of the retroperitoneal cyst (asterisks).

Upper panels (A-C) indicate T1-weighted images (T1WI) and lower panels (D-F) indicate T2-weighted images (T2WI). Note that signal intensity of the cyst content on T1WI becomes higher and that on T2WI becomes lower as the cyst becomes smaller.

At the age of 38 , the patient was referred to our hospital due to the relocation. At her initial visit, ultrasonography confirmed the presence of a unilocular cystic tumor measuring $15 \times 5 \mathrm{~cm}$ in the right upper abdomen. In MRI, the cyst content showed intermediate signal intensity on T1WI (Figure 1B) and high intensity on T2WI (Figure 1E). This signal pattern was consistent with mucus, allowing us to reach the diagnosis of primary retroperitoneal mucinous cystadenoma. In complying with the patient's intent, half-yearly follow-up was continued. At the age of 40 , the cystic tumor was found to have gradually shrunk, reaching to $10 \times 4 \mathrm{~cm}$ and the cyst content showed intermediate signal intensity both on T1WI and on T2WI (Figure 1C and 1F).

At the age of 41 , one year after her previous visit, sudden re-increase of the tumor size to $17 \times 14 \mathrm{~cm}$ was detected by ultrasonography. The patient complained of abdominal fullness. In MRI, emergence of three mural nodules measuring about $1 \mathrm{~cm}$ was also detected (Figure 2A). These mural nodules exhibited high signal intensity on diffusion-weighted imaging (DWI) (Figure 2B) and low value on apparent diffusion coefficient (ADC) mapping 
(Figure 2C), strongly suggesting that they were composed of malignant cells. No lymph node or organ metastasis was suspected in the enhanced thoraco-abdominal computed tomography (CT). The patient accepted to undergo surgical removal of the cystic tumor.
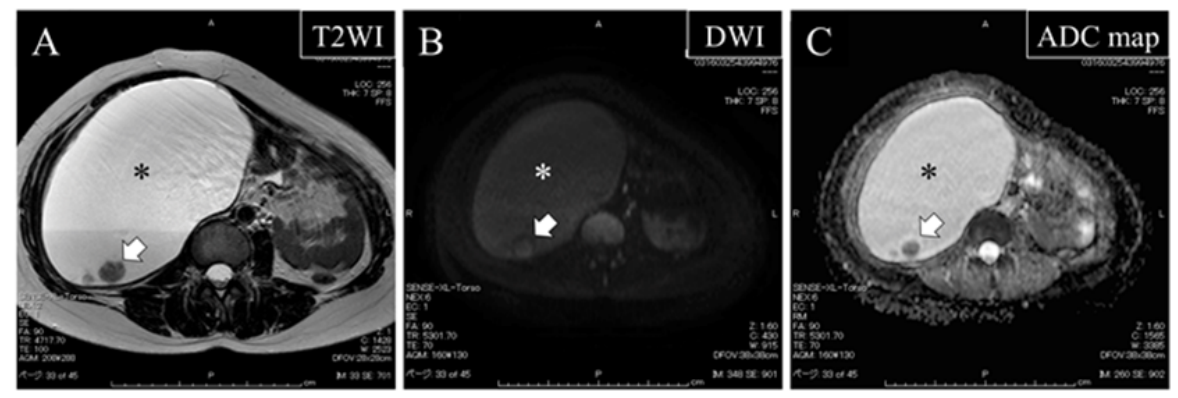

Figure 2: Magnetic resonance images at the age of 41 showing remarkable enlargement of the retroperitoneal cyst (asterisk) accompanied by the emergence of mural nodules (arrows)

(A) T2-weighted image (T2WI)

(B) Diffusion-weighted image (DWI)

(C) Apparent diffusion coefficient (ADC) map

Note that the mural nodules (arrows) exhibit high signal intensity on DWI (B) and low value on ADC map (C), strongly suggesting that they are composed of malignant cells.

At laparotomy, a cystic tumor of adult head size was vaulting the peritoneum and displacing the ascending colon medially (Figure 3A). The retroperitoneal tumor was covered by the outermost capsule of the ascending colon and was engaged in the right paracolic gutter. While the uterus was enlarged to over newborn head size due to multiple fibroids, bilateral fallopian tube and ovary appeared normal. The retroperitoneal tumor had no direct connection with the surrounding organs. Small amount of thin bloody ascites, which proved to be negative for malignancy in the intraoperative pathological consultation, was accumulated in the Douglas pouch. The tumor was carefully separated from the ascending colon and was successfully removed intact. The resected cystic tumor was opened in the operating room, exposing three fragile mural nodules on its inner surface. One of the mural nodules was submitted for the intraoperative pathological diagnosis. The nodule was mostly composed of necrotic tissue and was devoid of any obviously malignant cells. Since the patient had desired to preserve her fertility, a hysterectomy and salpingooophorectomy were withheld.

On gross examination, the resected specimen was a unilocular, smooth-surfaced, cystic tumor measuring $20 \mathrm{~cm}$ in diameter. The cyst contained dark-brownish mucin with papillary growth of three fragile nodules on its inner surface (Figure 3B). On histological examination, a benign single layer of mucin-producing high columnar epithelial cells lined most of the cystic wall. Although the protruded portions of the mural nodules exhibited extensive necrosis that 
did not allow for further histological evaluation, minimal invasion of atypical microglandular structures into the surrounding fibrous stroma was detected at their basal portions (Figure 3C). The immunohistochemical analysis showed that benign epithelial cells as well as adenocarcinoma cells were negative for calretinin (mesothelial marker) and were positive for cytokeratin 7 (epithelial marker) (Figure 3D). From these findings, the final pathological diagnosis was mucinous cytadenoma with focal microinvasive adenocarcinoma arising in the retroperitoneum.
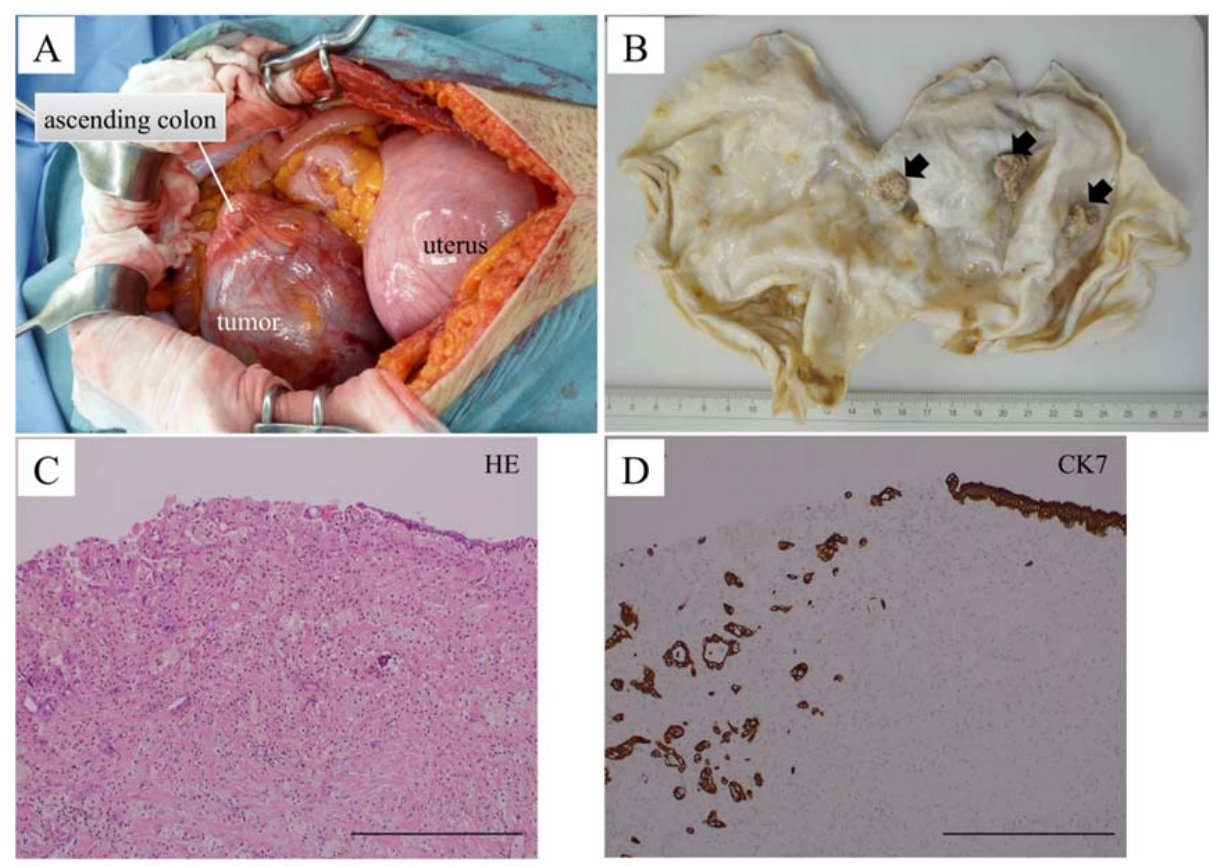

Figure 3: (A) Intraoperative photo showing the retroperitoneal tumor, uterus, and ascending colon. The cystic tumor is vaulting the peritoneum and displacing the ascending colon medially.

(B) Gross appearance of the opened cystic tumor after formalin fixation. Arrows indicate three mural nodules growing on the inner surface.

(C) Hematoxylin and eosin (HE) staining of the boundary region between benign mucinous epithelium and malignant microinvasive adenocarcinoma. Protruded necrotic portion of the mural nodule is exfoliated during the preparation of the section. Scale bar indicates $500 \mu \mathrm{m}$.

(D) Cytokeratin 7 (CK7) immunostaining of the same site as (C). Scale bar indicates $500 \mu \mathrm{m}$.

Postoperative recovery of the patient was uneventful. Since no residual malignant lesion was suspected in postoperative ${ }^{18} \mathrm{~F}$-fluorodeoxyglucose (FDG)-positron emission tomography (PET)/CT, additional treatment 
including secondary laparotomy or systemic chemotherapy was not offered. The patient is doing well without evidence of disease 12 months after the operation.

\section{Discussion}

The present case afforded an invaluable opportunity to observe the natural course of primary retroperitoneal mucinous tumor owing to the patient's unbending determination not to undergo an operation. In this case, the remarkable re-enlargement and emergence of three mural nodules suddenly took place in the retroperitoneal unilocular cyst after seven years of follow-up. These mural nodules were histologically composed of microinvasive mucinous adenocarcinoma focally scattered in the benign epithelial lining of mucinous cystadenoma. These findings strongly suggest that mucinous cystadenocarcinoma arose from the pre-existing benign mucinous cystadenoma. This is the first case report that lends evidence for the presence of adenoma-borderline tumor-carcinoma sequence in primary retroperitoneal mucinous tumor.

According to a meta-analysis by Myriokefalitaki et al., 73 female and 5 male cases of primary retroperitoneal mucinous cystadenocarcinoma had been reported by 2015 [12]. Median age at diagnosis was 42.0 years (range 1886 years), with women being significantly younger than men (42.0 years vs. 62.2 years, p=0.005). This apparent preponderance of premenopausal women suggests that certain specific milieu such as high estrogen concentration could contribute to its pathogenesis. Follow-up data were identified in 57 women (range 1-130 months, with a median of 15 months). During the follow-up period, 23 recurrences (40\%) with median time from diagnosis to recurrence of 8 months (range 1-58 months) and 14 deaths (25\%) were reported, illustrating relatively poor prognosis of this disease.

In the present case, no operative procedures other than tumor resection was executed because the patient had desired to preserve her fertility. In the same meta-analysis, the median survival period for the women who have undergone bilateral salpingo-oophorectomy is not significantly different from those who have not (53.6 \pm 4.2 months vs. 55.9 \pm 7.1 months, $\mathrm{p}=0.173$ ), suggesting that addition of bilateral salpingo-oophorectomy to simple tumor resection has no beneficial effect on the prognosis of primary retroperitoneal mucinous cystadenocarcinoma [12]. Therefore, at the present time, it is advisable that adjuvant bilateral salpingo-oophorectomy with or without hysterectomy should be restricted to postmenopausal women [13].

In the ovary, the response rate to platinum-based chemotherapy for invasive mucinous carcinoma is no more than $12.5 \%$ [14]. Considering the morphological similarity between ovarian and retroperitoneal mucinous carcinoma, it is not prudent to expect high response rate of chemotherapy against the retroperitoneal counterpart. In fact, the same meta-analysis failed to demonstrate any beneficial effect of adjuvant chemotherapy on the postoperative prognosis of primary retroperitoneal mucinous cystadenocarcinoma [12]. From these data, we believe that chemotherapy should be reserved for the patients with obvious residual lesion after the operation. In the preset case, since no residual lesion was suspected in the postoperative FDG-PET/CT, we did not offer adjuvant chemotherapy. 
Nevertheless, the presence of mural nodules in primary retroperitoneal mucinous cystadenocarcinoma has been reported to predict worse prognosis [15]. Considering that we were not able to extract any significant tumor marker elevated prior to the operation, close follow-up with regular systemic imaging should be warranted in the present case.

In MRI, the signal intensity of mucus varies depending on the degree of its mucin concentration, i.e., the signal intensity on T1WI becomes higher and that on T2WI becomes lower as the mucin gets thicker. This is in contrast with serous fluid, which almost invariably shows low signal intensity on T1WI and high intensity on T2WI. In the present case, the cyst content exhibited low T1WI signal and high T2WI signal in the initial MRI, leading to the wrong diagnosis of retroperitoneal Mullerian cyst, which was reported to contain serous fluid [11]. During the subsequent six years, however, the T1WI signal became higher and the T2WI signal became lower as the cyst has shrunk. Owing to this temporal change, which was considered to reflect the intracystic mucin condensation, we were able to reach the correct diagnosis of primary retroperitoneal mucinous tumor.

\section{Conflicts of interest}

The authors report no conflicts of interest. The authors alone are responsible for the content and writing of the paper.

\section{References}

1. Lachman MF, Berman MM. The ectopic ovary. A case report and review of the literature. Archives of pathology \& laboratory medicine 115 (1991): 233-235.

2. Williams PP, Gall SA, Prem KA. Ectopic mucinous cystadenoma. A case report. Obstetrics and gynecology 38 (1971): 831-837.

3. Banerjee R, Gough J. Cystic mucinous tumours of the mesentery and retroperitoneum: report of three cases. Histopathology 12 (1988): 527-532.

4. Fujii S, Konishi I, Okamura H, Mori T. Mucinous cystadenocarcinoma of the retroperitoneum: a light and electron microscopic study. Gynecologic oncology 24 (1986): 103-112.

5. Bell DA. Origins and molecular pathology of ovarian cancer. Modern pathology: an official journal of the United States and Canadian Academy of Pathology, Inc. 2005;18 Suppl 2:S19-32.

6. Dubeau L. The cell of origin of ovarian epithelial tumours. The Lancet Oncology 9 (2008): 1191-1197.

7. Yang DM, Jung DH, Kim H, Kang JH, Kim SH, et al. Retroperitoneal cystic masses: CT, clinical, and pathologic findings and literature review. Radiographics : a review publication of the Radiological Society of North America, Inc 24 (2004): 1353-1365.

8. Puls LE, Powell DE, DePriest PD, Gallion HH, Hunter JE, Kryscio RJ, et al. Transition from benign to malignant epithelium in mucinous and serous ovarian cystadenocarcinoma. Gynecologic oncology 47 (1992): 53-57. 
9. Riopel MA, Ronnett BM, Kurman RJ. Evaluation of diagnostic criteria and behavior of ovarian intestinaltype mucinous tumors: atypical proliferative (borderline) tumors and intraepithelial, microinvasive, invasive, and metastatic carcinomas. The American journal of surgical pathology 23 (1999): 617-635.

10. Ichikawa Y, Nishida M, Suzuki H, Yoshida S, Tsunoda H, et al. Mutation of K-ras protooncogene is associated with histological subtypes in human mucinous ovarian tumors. Cancer research 54 (1994): 33-35.

11. Lee J, Song SY, Park CS, Kim B. Mullerian cysts of the mesentery and retroperitoneum: a case report and literature review. Pathology international 48 (1998): 902-906.

12. Myriokefalitaki E, Luqman I, Potdar N, Brown L, Steward W, Moss EL. Primary retroperitoneal mucinous cystadenocarcinoma (PRMCa): a systematic review of the literature and meta-analysis. Archives of gynecology and obstetrics 293 (2016): 709-720.

13. Kessler TM, Kessler W, Neuweiler J, Nachbur BH. Treatment of a case of primary retroperitoneal mucinous cystadenocarcinoma: is adjuvant hysterectomy and bilateral salpingo-oophorectomy justified? American journal of obstetrics and gynecology 187 (2002): 227-232.

14. Shimada M, Kigawa J, Ohishi Y, Yasuda M, Suzuki M, Hiura M, et al. Clinicopathological characteristics of mucinous adenocarcinoma of the ovary. Gynecologic oncology 113 (2009): 331-334.

15. Kanayama T, Yoshino K, Enomoto T, Ohashi H, Fujita M, Ueda Y, et al. Primary retroperitoneal mucinous cystadenocarcinoma with mural nodules: a case report and literature review. International journal of clinical oncology 17 (2012): 407-411.

Citation: Atsuko Taga, Yukiyasu Sato, Shota Kanbayashi, Yasufumi Ri, Ikuko Emoto, Shunsuke Maruyama, Tomoyuki Shirase. Clinical Evidence for Adenoma-Carcinoma Sequence in Primary Retroperitoneal Mucinous Cystadenocarcinoma. Obstetrics and Gynecology Research 1 (2018): 001-008.

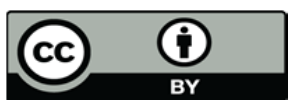

This article is an open access article distributed under the terms and conditions of the Creative Commons Attribution (CC-BY) license 4.0 